\title{
Identification of binding interactions between myeloperoxidase and its antibody using SERS
}

\author{
E. S. Papazoglou*, S. Babu, S. Mohapatra, D. R. Hansberry and C. Patel
}

Surface Enhanced Raman Spectroscopy (SERS) is a widely used spectroscopic method that can dramatically increase the sensitivity of Raman spectroscopy and has demonstrated significant benefit in the identification of biological molecules. We report the use of SERS in differentiating the bound immunocomplex of myeloperoxidase (MPO) and its antibody from the unbound complex and its individual components. The SERS signal was enabled by gold nanoparticles attached to MPO, pAb and their immunocomplex at an excitation wavelength of $785 \mathrm{~nm}$. The obtained SERS spectrum of MPO is in agreement with previous literature. Comparative SERS spectrum analysis of MPO, pAb, and their immunocomplex reveals the significant peak shifts and intensity variations caused by the conformational changes due to the immunocomplex formation. Several key areas have been identified which correspond to specific amino acids being shielded from undergoing resonance while new amino acid residues are made visible in the SERS spectrum of the immunocomplex and could be a result of conformational binding. Our work demonstrates the capability of SERS to identify binding events and differentiate an immunocomplex from its unbound components with direct applications in biosensors.

Keywords: Gold nanoparticle; Antigen; Immunocomplex; Biosensors

Citation: E. S. Papazoglou, S. Babu, S. Mohapatra, D. R. Hansberry and C. Patel, "Identification of binding interactions between myeloperoxidase and its antibody using SERS”, Nano-Micro Lett. 2, 74-81 (2010). doi:10.5101/nml.v2i2. p74-82

Raman spectroscopy is a useful technique, based on Raman scattering, that has been widely used for molecular material characterization. When the electric field of a monochromatic light source (laser) interacts with a molecule it induces a dipole moment and causes the molecule to deform. The oscillatory nature of the laser beam's electric field causes periodic changes in the dipole moment resulting in vibration of the molecule which are characteristic of the molecule under investigation. The interaction of the molecule with a photon results in the molecule, either losing one vibrational quantum of energy or gaining one vibrational quantum of energy, and this is referred to as Stokes Raman scattering or anti-Stokes Raman scattering, respectively. Raman spectroscopy most commonly measures vibrational energy states but can also measure rotational or electronic energy states. The main challenge of Raman spectroscopy has been the difficulty in separating the weak inelastic Raman scattering signal from the overwhelmingly dominant elastic Rayleigh scattering. For every photon that undergoes Raman scattering there are at least 10 million photons that undergo Rayleigh scattering.

Surface-enhanced Raman spectroscopy (SERS) provides a solution to this challenge by enhancing the signal intensity of Raman scattering of molecules adsorbed to roughened metal surfaces by as much as $10^{15}$ [1]. Fleischman et al. [2] was the first to note this enhanced effect in 1973 while observing the adsorption of pyridine on a silver electrode. Later two groups, 
Jeanmarie et al. [3] and Albrecht et al. [4] independently realized this as a unique phenomenon and proposed both an electromagnetic mechanism and a chemical mechanism, as theoretical explanations for the enhanced signal. Gold and silver nanoparticles are commonly used as SERS substrates with gold particles being used more frequently due to their high sensitivity and stability compared to silver compounds [5-8]. It must be noted that the enhanced Raman signal enabled by the plasmon resonance of gold and silver nanoparticles gave rise to new biosensors that have been used for identifying the binding region in proteins [9], and for studying binding affinity of immunoreactions [10]. Signal analysis of SERS was used to identify the native constituents of live epithelial cells employing endocytosed $60 \mathrm{~nm}$ gold nanoparticles [11]. SERS has also been used to differentiate bacteria from bacteriophages by conjugating them to $60 \mathrm{~nm}$ gold nanoparticles [12] and in single molecule detection $[13,14]$. The combination of plasmon resonance based sensing with real-time SERS analysis could become a novel tool for interrogating the dynamics of protein binding interactions.

Applications of SERS in immunosensing include the successful detection of the thyroid stimulating hormone (TSH) [15], monitoring the immunocomplex formation between mouse $\operatorname{IgG}$ and goat anti-mouse $\operatorname{IgG}$ [16], detection of membrane bound enzymes within cells and correlation of prostaglandinH-synthase (PGHS) antigen levels [17], and detection of conformational binding of anti-mouse IgG (bound to gold nanoparticles of $29.7 \mathrm{~nm}$ diameter) to mouse IgG antigen [18].

Myeloperoxidase (MPO) is a lysosomal protein found in neutrophilic granulocytes, often overexpressed in inflammatory diseases [19-21]. MPO is a vital protein found in neutrophilic granulocytes that has an instrumental role in attacking bacteria and foreign pathogens. Neutrophilic granulocytes phagocytose pathogens and eliminate them through chemical reactions. MPO is capable of producing both hypochlorous acid and tyrosyl radicals in independent pathways. Hypochlorous acid and the tyrosyl radical are both cytotoxic and degrade bacteria and foreign pathogens. MPO is a $140 \mathrm{kDa}$ dimmer composed of two identical halves, each with a covalently bound heme and connected by a lone disulfide bond. In addition to the heme group located on each half of the MPO molecule there is a bound calcium ion and three Asn-linked glycosylations (at Asn189, Asn225, Asn317) [22]. Sibbet et al. [23] examined the structure of canine MPO using resonance Raman spectroscopy and concluded that MPO contains two equivalent chlorine prosthetic groups.

To our knowledge Raman spectroscopic studies on MPO have been primarily performed using Resonance Raman
Spectroscopy (RRS). RRS yields higher peak intensity compared to conventional Raman spectroscopy which translates to a lower sample concentration requirement. Our interest lies in using SERS to identify bands that are unique to the immunocomplex of MPO and its antibody and explore the use of such bands as signatures of binding events. In this paper, we report our results in differentiating the SERS signal of the $\mathrm{MPO} / \mathrm{pAb}$ immunocomplex from the unbound complex and its individual components.

\section{EXPERIMENTAL METHODS}

All chemicals were purchased from Sigma Aldrich unless otherwise mentioned, pAb (rabbit anti human myeloperoxidase) was purchased from ABD-Serotec and myeloperoxidase (MPO) was purchased from Lee Biosolutions Inc.

Gold nanoparticles were prepared according to Frens, G. [24] with added modifications. $500 \mu 1$ of $1 \%$ chloroauric acid $\left(\mathrm{HAuCl}_{4}\right)$ were added to $50 \mathrm{ml}$ of distilled $\mathrm{H}_{2} \mathrm{O}$ and heated to a boil under constant stirring. Upon boiling $400 \mu 1$ of $1 \%$ citric acid $\left(\mathrm{C}_{6} \mathrm{H}_{8} \mathrm{O}_{7}\right)$ were added. The size of nanoparticles could be controlled by varying the volume of citric acid being added; higher volume corresponds to smaller particles and lower volume corresponds to larger particles. $400 \mu 1$ of $1 \%$ citric acid correspond to $40 \mathrm{~nm}$ particles. The solution was refluxed until the color was changed from dark blue to red. The solution was then removed from heat and allowed to cool to room temperature. The solution was further dialyzed in $\mathrm{DI} \mathrm{H}_{2} \mathrm{O}$ for 48 hours with the water being changed at 3,12 , and 24 hours, in order to remove citrate ions from the solution. Particle characterization was performed with UV-vis spectroscopy [25], atomic force microscopy, and scanning electron microscopy. Thus prepared gold nanoparticles were then immobilized on silane functionalized glass slides. Silanization of glass slides was achieved by the method followed by Park et al. [26]. In brief, glass slides were cleaned using piranha solution $(1: 3 \mathrm{v} / \mathrm{v}$, $\mathrm{H}_{2} \mathrm{O}_{2}: \mathrm{H}_{2} \mathrm{SO}_{4}$ ) and dried under nitrogen. Cleaned glass slides were then immersed in 3\% 3-aminopropyltrimethoxysilane (APTMS) in methanol for 3 hours. Silanized slides were then rinsed thoroughly with methanol followed by DI water and drying with a jet of dry nitrogen. Silanized glass slides were then immersed in the dialyzed gold nanoparticle solution for 3 hours. Slides with immobilized gold nanoparticles were then washed with DI water and air dried. A well with a capacity of $\sim 100 \mathrm{~mL}$ was constructed using plastic pipette and epoxy on top of the glass slide to facilitate $\mathrm{pAb}$ immobilization and subsequent SERS data collection. 
Polyclonal antibody was immobilized by letting $\sim 100 \mathrm{ml}$ of $100 \mathrm{nM}$ pAb solutions interact with the gold nanoparticle coated slides for 15 minutes at room temperature followed by thorough washing with 1x PBS buffer (pH7.4). Gold nanoparticles coated with pAb were then allowed to interact with MPO $(100 \mu 1,1 \mu \mathrm{M})$ for 15 minutes at room temperature followed by washing. A similar procedure was followed to immobilize MPO (100 $\mu 1,1$ $\mathrm{M})$ directly on gold nanoparticles. SERS data was collected from the Au-pAb conjugates, Au-pAb/MPO immunocomplex, and Au-MPO conjugates immediately after preparation using a Renishaw RM1000 confocal Raman microspectrometer with a 50x long focal microscope. A $785 \mathrm{~nm}$ diode laser $(15 \mathrm{~mW})$ was used to collect the SERS signal. Care was taken to maintain a $\sim 50 \mu \mathrm{l}$ of PBS buffer to minimize thermal damage and the SERS data was collected from an area of $\sim 40 \mathrm{x} 40 \mu \mathrm{m}$ square $(\sim 100$ data points) located at the center of the well. SERS signal from glass slides coated with gold nanoparticles was also collected and served as the background signal.

Spectra with errors due to cosmic ray influence were removed manually resulting in an average of 75 spectra per sample. Individual spectra were then subjected to a three point baseline correction. The three points for baseline correction were kept the same for all spectra and samples, typically the first and last point corresponding to the beginning and end of the wave

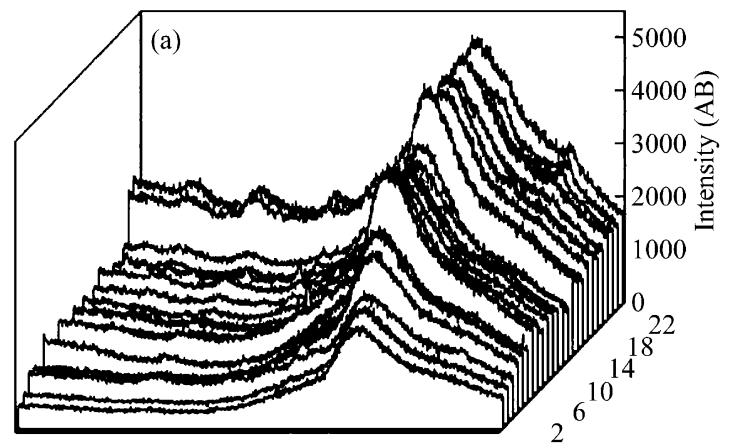

$600 \quad 800 \quad 1000 \quad 1200 \quad 1400 \quad 1600$

Wavenumber $\left(\mathrm{cm}^{-1}\right)$

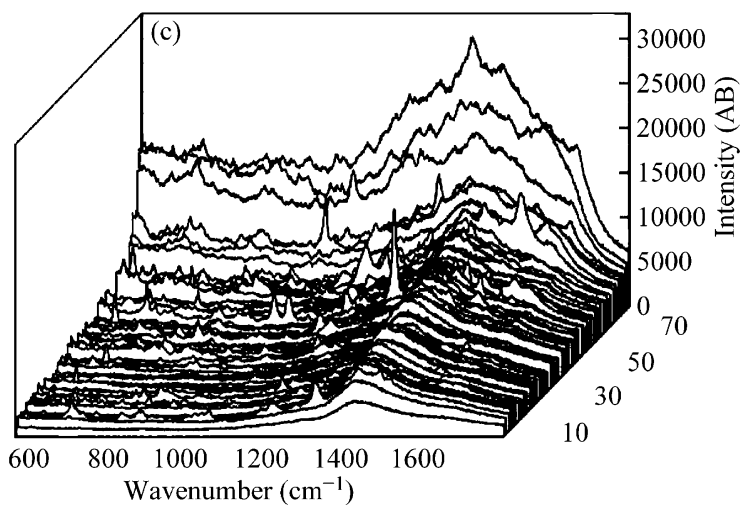

FIG. 1. Raw SERS spectra of (a) Gold nanoparticles, (b) polyclonal antibody, (c) myeloperoxidase and (d) immunocomplex.
This baseline correction applied to all data sets helped smooth the data and allow an appropriate comparison. Following the baseline correction individual spectra were normalized and averaged. In order to remove the gold nanoparticle (AuNp) signature from the spectra, the average AuNp spectrum set was subtracted from averaged spectra of Ab, MPO and MPO-pAb. The sum of $\mathrm{Ab}$ and MPO spectra was obtained by simply averaging the two spectra in Origin Pro. Origin 8.0's peak analyzer module was used to fit peaks to the averaged spectra.

\section{RESULTS AND DISCUSSION}

Raw SERS spectra of AuNp, Ab, MPO, and MPO-pAb are shown in Fig. 1a-1d, respectively. Individual spectra in Fig. 1 were sorted by their average intensity for easy visualization. The background spectra i.e. spectra of AuNp (see Fig. 1a) reveal the typical fluorescence response of AuNp under experimental conditions [27]. Peak positions as well as peak width remain the same between various spots on the scanned area, demonstrating the reproducibility of the collected SERS data. Figure 1b-1d demonstrate the influence of the AuNp on the spectra of the pAb, MPO and MPO-pAb respectively. This necessitates removal of the AuNp interference from the rest of the dataset. Baseline corrected Ab, MPO and MPO-pAb spectra are shown in Fig. 2a, $\mathrm{b}$ and $\mathrm{c}$, respectively. In order to identify spectral changes due to
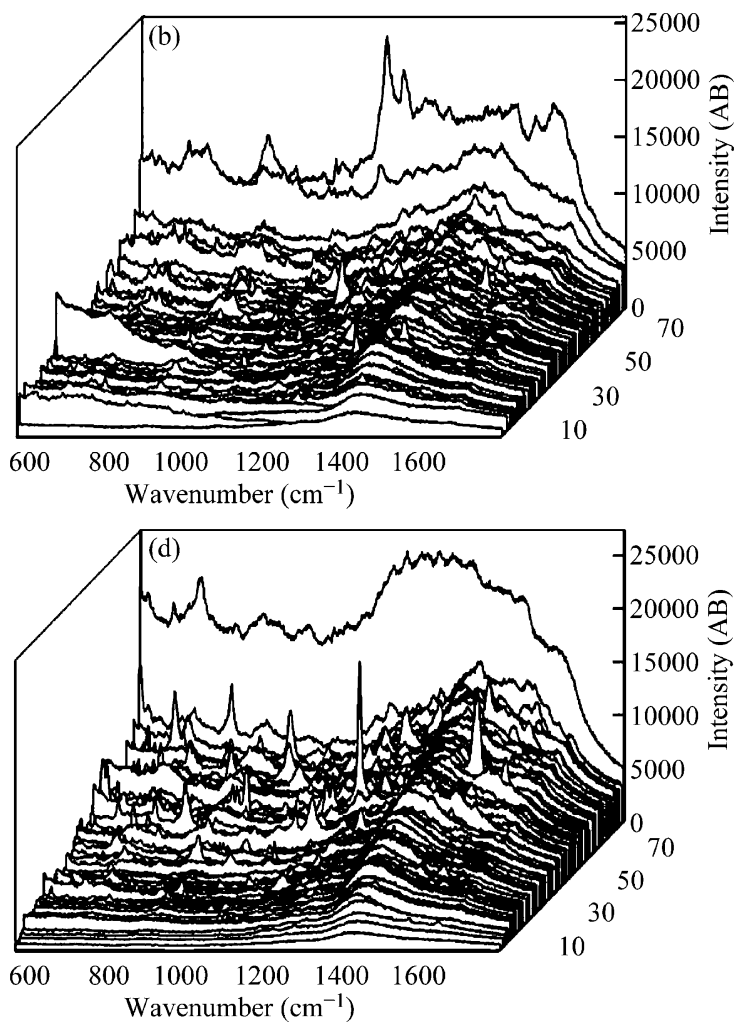

the binding of $\mathrm{pAb}$ and MPO as opposed to simple superposition numbers of the spectra and the third point being set at $1369 \mathrm{~cm}^{-1}$. 
we compared the spectra of AbMPO and the sum of spectra of $\mathrm{pAb}$ and MPO as shown in Fig. 3. Table 2 the peak positions of AbMPO along with the peaks that were found to be distinct for their immunocomplex. The peak positions that are unique for the immunocomplex are shown with the respective error bars (average of 80 spectra).

The SERS spectrum of MPO (see Fig. 2b) exhibits strong similarities to the Raman signals reported in previous studies [23,28-31]. It is relatively weak below $1100 \mathrm{~cm}^{-1}$, besides three small peaks between $646 \mathrm{~cm}^{-1}\left(v_{48} / v_{25}\right)$ to $684 \mathrm{~cm}^{-1}\left(v_{7}\right)$, at 835
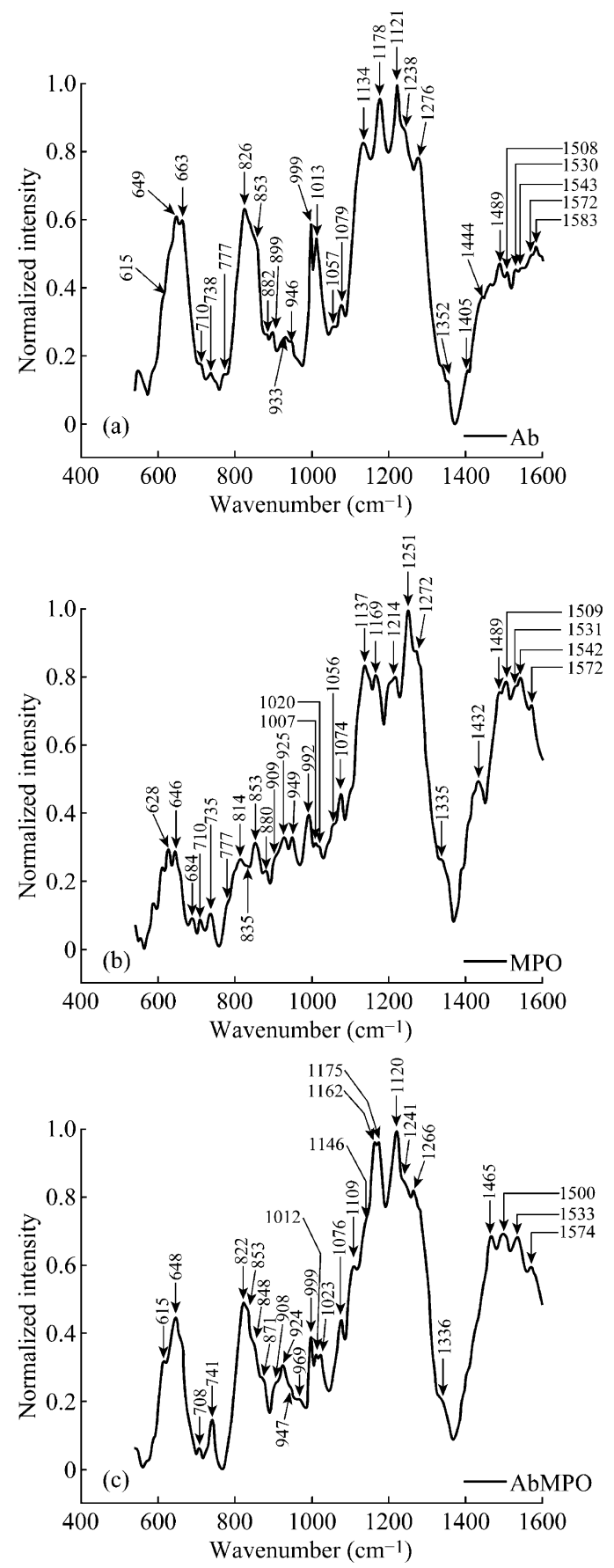

FIG. 2. SERS spectra of averaged (a) Ab, (b) MPO, and (c) AbMPO

$\mathrm{cm}^{-1}$, and between $992 \mathrm{~cm}^{-1}(\gamma(\mathrm{CH}))$ and $1007 \mathrm{~cm}^{-1}\left(v_{45}\right)$. Table 1
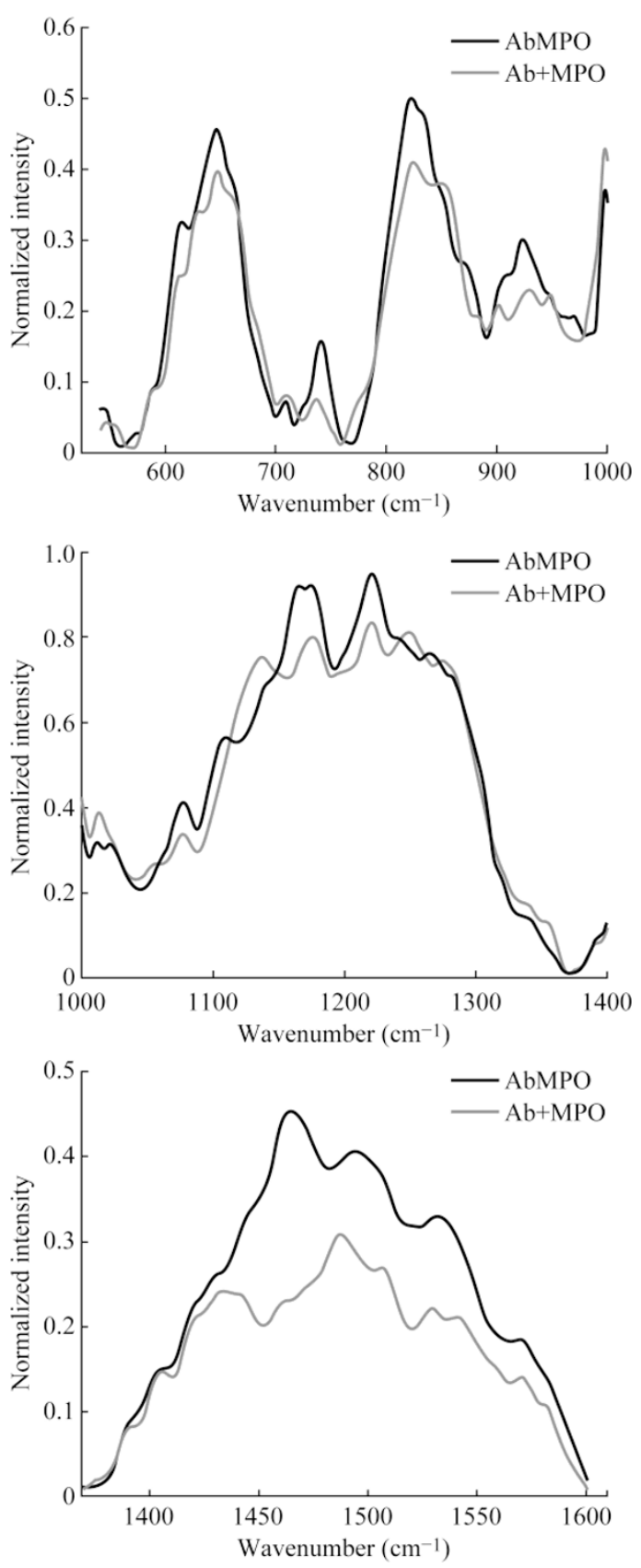

FIG. 3. Comparison of AbMPO spectra with sum of $A b$ and MPO spectra.

summarizes the MPO Raman peaks published by various studies. The minor peak assignments in our study are in excellent agreement with Zbylut S. D. et al., including the vibrational modes at $v_{45}\left(1007 \mathrm{~cm}^{-1}\right), \gamma(\mathrm{CH})\left(992 \mathrm{~cm}^{-1}\right), v_{46}\left(925 \mathrm{~cm}^{-1}\right), \gamma_{10}$ $\left(853 \mathrm{~cm}^{-1}\right), \gamma_{15}\left(710 \mathrm{~cm}^{-1}\right), v_{7}\left(684 \mathrm{~cm}^{-1}\right)$, and $v_{48} / v_{25}\left(646 \mathrm{~cm}^{-1}\right)$ [32]. The MPO spectrum from $1120 \mathrm{~cm}^{-1}$ to $1260 \mathrm{~cm}^{-1}$ is also found to be similar to that previously published from resonance Raman spectra [31]. Notably there are significant peaks at 1137 $\mathrm{cm}^{-1}\left(v_{44}\right), 1214 \mathrm{~cm}^{-1}\left(v_{13}\right)$, and $1251 \mathrm{~cm}^{-1}\left(v_{42}\right)$. These peaks are consistent with previous literature on resonance Raman spectra of MPO [23,28-31]. Our work using a $785 \mathrm{~nm}$ excitation 
Table 1. Comparison of MPO Raman signal across multiple studies.

\begin{tabular}{|c|c|c|c|c|c|c|c|c|c|c|c|c|c|}
\hline $\begin{array}{l}\text { vibrational } \\
\text { mode }\end{array}$ & $\begin{array}{c}\text { sym. } \\
\text { species }\end{array}$ & $\begin{array}{c}\mathrm{MPO}^{3+} \\
\mathrm{a}\end{array}$ & $\begin{array}{c}\mathrm{MPO}^{3+} \\
\mathrm{b}\end{array}$ & $\begin{array}{c}\mathrm{MPO}^{2+} \\
\text { b }\end{array}$ & $\begin{array}{c}\mathrm{MPO}^{3+} \\
\text { c }\end{array}$ & $\begin{array}{c}\mathrm{MPO}^{2+} \\
\text { c }\end{array}$ & $\begin{array}{c}\mathrm{MPO}^{3+} \\
\mathrm{d}\end{array}$ & $\begin{array}{c}\mathrm{MPO}^{2+} \\
\mathrm{d}\end{array}$ & $\begin{array}{c}\mathrm{MPO}^{3+} \\
\mathrm{e}\end{array}$ & $\begin{array}{c}\mathrm{MPO}^{3+} \\
\mathrm{f}\end{array}$ & $\begin{array}{c}\mathrm{MPO}^{3+} \\
\mathrm{g}\end{array}$ & $\begin{array}{c}\mathrm{MPO}^{3+} \\
\mathrm{h}\end{array}$ & $\begin{array}{c}\mathrm{MPO}^{2+} \\
\mathrm{h}\end{array}$ \\
\hline$v 10$ & $B 1 g$ & - & $1620 \mathrm{~s}$ & $1608 \mathrm{~s}$ & 1617 & - & - & - & - & - & - & - & - \\
\hline$v(C=C)$ & - & - & $1615 \mathrm{~s}$ & overlapped & - & 1607 & 1614 & 1606 & 1614 & 1612 & 1614 & 1610 & 1607 \\
\hline v37 & $E u$ & - & $\begin{array}{c}1595 \mathrm{j}, \mathrm{k} \\
\mathrm{m}\end{array}$ & - & 1588 & - & 1592 & 1582 & - & - & 1593 & - & - \\
\hline v2 & $A 1 g$ & - & $1590 \mathrm{~s}$ & $1588 \mathrm{~s}$ & 1568 & 1587 & - & - & 1585 & 1587 & 1576 & 1585 & 1586 \\
\hline$v 19$ & $A 2 g$ & 1572 & $1569 \mathrm{~m}$ & $1564 \mathrm{i}$ & - & 1561 & - & - & - & - & - & - & - \\
\hline v11 & $B 1 g$ & - & $1552 \mathrm{~s}$ & $1548 \mathrm{~m}$ & 1551 & 1544 & - & - & - & 1550 & 1543 & 1550 & 1549 \\
\hline- & - & - & $1545 \mathrm{j}$ & - & - & - & 1552 & 1545 & - & - & - & - & - \\
\hline v38 & $E u$ & 1531 & $1525 \mathrm{~m}$ & $1527 \mathrm{i}$ & 1523 & 1524 & 1523 & 1523 & - & 1523 & - & 1524 & 1528 \\
\hline $2 v 15$ & - & 1509 & $1505 \mathrm{j}$ & - & - & - & - & - & - & - & 1504 & - & - \\
\hline v3 & $A 1 g$ & 1488 & $1483 \mathrm{~m}$ & $1470 \mathrm{~m}$ & 1482 & - & 1486 & 1472 & - & 1479 & 1481 & 1481 & - \\
\hline$v 28$ & $B 2 g$ & - & 14741 & - & - & 1469 & - & - & 1472 & - & - & - & 1472 \\
\hline$\delta(=\mathrm{CH} 2)$ & - & 1432 & $1430 \mathrm{w}$ & $1425 \mathrm{w}$ & - & 1422 & - & 1424 & - & - & - & - & 1424 \\
\hline$v 40$ & $E u$ & - & $\begin{array}{c}1396 \\
\text { sh }\end{array}$ & $1401 \mathrm{sh}$ & - & 1396 & - & - & - & - & - & - & - \\
\hline$v 20$ & $A 2 g$ & - & 13871 & $1379 \mathrm{w}$ & 1378 & - & - & - & - & - & 1386 & - & - \\
\hline$v 12$ & $B 1 g$ & - & $1375 \mathrm{~s}$ & $1362 \mathrm{sh}$ & - & 1378 & - & - & - & 1377 & - & 1376 & 1377 \\
\hline v4 & $A 1 g$ & - & $1366 \mathrm{~s}$ & $1352 \mathrm{~s}$ & 1366 & - & 1366 & 1359 & - & - & - & - & - \\
\hline $2 v 7$ & - & - & $1362 \mathrm{j}$ & - & - & 1351 & - & - & 1359 & 1363 & 1363 & 1362 & 1355 \\
\hline$v 41$ & $E u$ & 1335 & $1335 \mathrm{~m}$ & $1331 \mathrm{w}$ & 1339 & 1329 & - & - & - & - & 1332 & 1331 & - \\
\hline$\delta(\mathbf{C H}=)$ & - & - & $\begin{array}{c}1326 \\
\text { sh }\end{array}$ & $1320 \mathrm{sh}$ & - & - & - & - & - & - & - & - & - \\
\hline$v 21$ & $A 2 g$ & - & $1305 \mathrm{~m}$ & $1305 \mathrm{w}$ & 1307 & 1306 & - & - & - & 1307 & 1307 & 1306 & - \\
\hline CH2 wag & - & 1272 & $1268 \mathrm{~m}$ & $1267 \mathrm{~m}$ & 1267 & 1267 & - & - & - & - & - & 1265 & 1265 \\
\hline$v 42$ & $E u$ & 1251 & $1248 \mathrm{w}$ & $1242 \mathrm{~m}$ & - & 1241 & - & - & - & - & 1238 & 1240 & 1242 \\
\hline CH2 twist & - & - & $1223 \mathrm{w}$ & - & - & - & - & - & - & - & - & - & - \\
\hline$v 13$ & $B 1 g$ & 1214 & $1205 \mathrm{~m}$ & $1205 \mathrm{~m}$ & 1207 & 1206 & - & - & - & 1206 & 1208 & 1205 & 1207 \\
\hline v30 & $B 2 g$ & 1169 & $\begin{array}{l}1173 \\
\text { vw }\end{array}$ & $1156 \mathrm{vw}$ & 1161 & - & - & - & - & - & - & 1163 & 1162 \\
\hline$v 44$ & $E u$ & - & $1142 \mathrm{k}$ & - & - & 1139 & 1116 & 1116 & - & - & - & - & - \\
\hline$v 14$ & $B 1 g$ & 1137 & $1130 \mathrm{~m}$ & $1137 \mathrm{w}$ & 1133 & - & - & - & 1131 & 1134 & 1130 & 1132 & 1138 \\
\hline$v 5 / v 22$ & $A 1 g / A 2 g$ & - & $1112 \mathrm{~m}$ & $1107 \mathrm{~m}$ & 1114 & 1107 & - & - & - & 1111 & 1111 & 1106 & 1107 \\
\hline$\delta(=\mathrm{CH} 2)_{\mathrm{as}}$ & - & 1073 & 1063 w & - & - & 1068 & - & - & - & - & - & 1069 & 1068 \\
\hline$v 23$ & $A 2 g$ & - & 1033 w & 1029 vw & 1034 & 1026 & - & - & - & - & 1034 & 1029 & 1027 \\
\hline$v 45$ & $E u$ & 1007 & $998 \mathrm{~m}$ & $984 \mathrm{w}$ & 1003 & - & - & - & - & 1003 & 1001 & 1003 & - \\
\hline$\gamma(\mathbf{C H})$ & - & 992 & $985 \mathrm{w}$ & 984 w & 979 & 989 & - & - & - & - & 984 & - & 983 \\
\hline$v 46$ & $E u$ & - & $935 \mathrm{vw}$ & - & 934 & - & - & - & - & - & - & - & - \\
\hline$\gamma\left(-\mathrm{C}_{\mathrm{b}} \mathbf{H}_{2}\right)_{\mathrm{s}}$ & - & 925 & - & - & - & 925 & - & - & - & - & - & - & - \\
\hline$\gamma 10$ & $B 1 u$ & 853 & $861 \mathrm{w}$ & - & 858 & 860 & - & - & - & - & - & 859 & 862 \\
\hline- & - & 835 & $830 \mathrm{w}$ & - & 835 & 825 & - & - & - & - & 832 & 837 & 841 \\
\hline$v 47$ & $E u$ & - & $770 \mathrm{sh}$ & - & - & - & - & - & - & - & - & - & - \\
\hline
\end{tabular}




\begin{tabular}{|c|c|c|c|c|c|c|c|c|c|c|c|c|c|}
\hline$v 15$ & $B 1 g$ & - & $757 \mathrm{~m}$ & $750 \mathrm{~m}$ & 757 & 748 & - & - & 748 & 757 & 757 & 749 & 749 \\
\hline$\gamma 11$ & $B 1 u$ & 710 & $717 \mathrm{~m}$ & $715 \mathrm{vw}$ & - & 717 & - & - & - & - & - & 715 & 715 \\
\hline$\gamma 15$ & $B 2 u$ & - & $704 \mathrm{sh}$ & $703 \mathrm{sh}$ & - & - & - & - & - & - & - & - & - \\
\hline v7 & $A 1 g$ & 684 & $675 \mathrm{~m}$ & $675 \mathrm{~m}$ & 676 & 675 & 676 & 676 & 676 & 675 & - & 675 & 676 \\
\hline v48/v25 & $E u / A 2 g$ & 646 & $562 \mathrm{w}$ & - & - & - & - & - & - & - & - & 557 & - \\
\hline$\gamma 21$ & $E g$ & - & - & - & - & - & - & - & - & - & - & - & - \\
\hline$v 49$ & $E u$ & - & $523 \mathrm{sh}$ & - & - & 520 & - & - & - & - & - & 517 & 517 \\
\hline$\gamma 12$ & $B 1 u$ & - & $506 \mathrm{~m}$ & - & - & - & - & - & - & - & - & - & - \\
\hline v33 & $B 2 g$ & - & $453 \mathrm{sh}$ & - & 443 & - & - & - & - & - & - & - & - \\
\hline$\gamma 22$ & $E g$ & - & $434 \mathrm{~m}$ & - & - & - & - & - & - & 437 & - & 437 & 438 \\
\hline$\delta\left(C_{\beta} C_{a} C_{b}\right)$ & - & - & $410 \mathrm{~s}$ & $403 \mathrm{vw}$ & 411 & 402 & 410 & - & - & 408 & - & 409 & 403 \\
\hline v8 & $A 1 g$ & - & $340 \mathrm{sh}$ & - & - & - & - & - & 344 & - & - & - & - \\
\hline$\gamma 6$ & $A 2 u$ & - & $328 \mathrm{~m}$ & $325 \mathrm{vw}$ & 329 & 326 & - & - & - & 328 & - & 329 & 324 \\
\hline v17 & $B 1 g$ & - & $313 \mathrm{j}$ & - & - & - & - & - & - & - & - & - & - \\
\hline
\end{tabular}

a, our data (excitation wavelength of $785 \mathrm{~nm}$ ); b, from [35] with additions from [32] (excitation wavelength of $406.7 \mathrm{~nm}$ ); $\mathrm{c}$, from [28] (excitation wavelength of 457.9 $\mathrm{nm}$ ); d, from [31] (excitation wavelength of $413.1 \mathrm{~nm}$ ); e, from [31] (excitation wavelength of $413.1 \mathrm{~nm}$ ); f, from [31] (excitation wavelength of $457.9 \mathrm{~nm}$ ); g, from [29] (excitation wavelength of 660nm); h, from [23] (excitation wavelength of $454.5 \mathrm{~nm}$ ); i, from [35], strong (excitation wavelength of 496.5 nm); j, from [35], observed or very strong in the spectrum (excitation wavelength of $568.2 \mathrm{~nm}$ ); $\mathrm{k}$, from [35], strong (excitation wavelength of $406.7 \mathrm{~nm}$ ); 1, from [35], strong (excitation wavelength of $514.5 \mathrm{~nm}$ ); m, medium; s, strong; sh, sharp, w, weak; vw, very weak.

Table 2. Comparison of SERS peak positions

\begin{tabular}{|c|c|c|c|c|c|c|c|c|c|}
\hline No & $\mathbf{A b}$ & MPO & $\begin{array}{c}\text { AbMPO } \\
\text { immunocomplex }\end{array}$ & $\begin{array}{l}\text { Ab }+ \\
\text { MPO }\end{array}$ & No & $\mathbf{A b}$ & MPO & $\begin{array}{c}\text { AbMPO } \\
\text { immunocomplex }\end{array}$ & $\begin{array}{l}\mathbf{A b}+ \\
\text { MPO }\end{array}$ \\
\hline 1 & 615 & & 615 & & 28 & & & 1109 & \\
\hline 2 & & 628 & & & 29 & 1134 & 1137 & & 1135 \\
\hline 3 & 649 & 646 & 648 & 649 & 30 & & & 1146 & \\
\hline 4 & 663 & & 661 & & 31 & & 1169 & 1162 & \\
\hline 5 & & 684 & & & 32 & 1178 & & 1175 & 1175 \\
\hline 6 & 710 & 710 & & 712 & 33 & 1221 & 1214 & 1220 & 1218 \\
\hline 7 & 738 & 735 & 741 & 735 & 34 & 1238 & & & \\
\hline 8 & 776 & & & 779 & 35 & & 1251 & & 1248 \\
\hline 9 & & 814 & & & 36 & 1276 & 1272 & 1266 & 1275 \\
\hline 10 & 826 & & 822 & 824 & 37 & & 1335 & & \\
\hline 11 & & 835 & 835 & & 38 & 1352 & & & \\
\hline 12 & 853 & 853 & 848 & 855 & 39 & 1405 & & & \\
\hline 13 & & & 871 & & 40 & 1444 & & & 1442 \\
\hline 14 & & 880 & & & 41 & & 1432 & & \\
\hline 15 & 882 & & & & 42 & & & 1465 & \\
\hline 16 & 899 & & & 902 & 43 & 1472 & & & \\
\hline 17 & & 925 & 924 & 925 & 44 & 1489 & 1488 & & 1489 \\
\hline 18 & 933 & & & & 45 & & & 1500 & \\
\hline 19 & 946 & 949 & & 950 & 46 & 1508 & 1509 & & 1508 \\
\hline 20 & & & 969 & & 47 & 1530 & 1531 & 1533 & 1532 \\
\hline 21 & & & 984 & & 48 & 1543 & & & 1543 \\
\hline 22 & 999 & 992 & 999 & 993 & 49 & & & & 1556 \\
\hline 23 & & 1007 & 1012 & 1011 & 50 & 1572 & 1572 & 1574 & 1573 \\
\hline 24 & 1013 & & & & 51 & 1583 & & & \\
\hline 25 & & 1020 & 1023 & & 52 & 1608 & & & 1602 \\
\hline 26 & 1057 & 1056 & & 1064 & 53 & & 1690 & & \\
\hline 27 & 1079 & 1074 & 1076 & & & & & & \\
\hline
\end{tabular}


used different excitation wavelengths (ranging from $406.7 \mathrm{~nm}$ to $660 \mathrm{~nm}$ ) [23,28-31]. Therefore the results obtained suggest that the key identifying MPO peaks are independent of the excitation wavelength, or the Raman method (RRS, CARS or SERS) that was used to collect the data. It is encouraging to also see that the SERS spectrum of the $\mathrm{pAb}$ has characteristic peaks similar to previously obtained Raman signals of an IgG. This is not surprising given the structural similarities of the antibodies. The complete list of peaks from Fig. 2 is presented in Table 2.

Figure 2 compares the SERS spectra of MPO, pAb, and the immunocomplex of MPO bound to pAb and Table 2 lists and compares the peaks of each spectrum. Beginning at $500 \mathrm{~cm}^{-1}$ and working towards $1650 \mathrm{~cm}^{-1}$ we observe distinct peak shifts, altered intensities, and unique peaks when comparing MPO, pAb, and their immunocomplex. Signal intensity alone may not be sufficient to differentiate between bimolecular especially in the SERS mode (amplification factors and specific binding may alter the signal intensity significantly) [33]. It may rather be a combination of signal intensity and peak position that can provide a more reliable means for identifying a given sample. The differences shown in Fig. 2 suggest that the immunocomplex formation could result in changes in conformation, orientation of bonds, and changes in the functional groups within the plasmon resonance distance of thegold nanoparticle. New peaks at $871 \mathrm{~cm}^{-1}, 1109 \mathrm{~cm}^{-1}$, and $1465 \mathrm{~cm}^{-1}$ present in the immunocomplex and not found in either MPO or pAb alone, indicate a tryptophan moiety made now visible in the SERS spectrum, possibly a result of conformational changes after binding. Furthermore, new peaks at $969 \mathrm{~cm}^{-1}, 984$ $\mathrm{cm}^{-1}, 1146 \mathrm{~cm}^{-1}$ and $1500 \mathrm{~cm}^{-1}$ which are not found in the MPO or pAb signal, indicate the immunocomplex formation. In the Tyr and Amide III region, a peak shift in the pAb spectra from $1276 \mathrm{~cm}^{-1}$ to $1266 \mathrm{~cm}^{-1}$ in the immunocomplex may also correspond to conformational changes.

The critical element in claiming that SERS is capable of identifying binding between MPO and the pAb is the significant difference between a composite spectrum obtained by addition of MPO and pAb and the SERS of the immunocomplex. For the purpose of comparison we are including the spectra of the immunocomplex and the composite spectrum (i.e. sum of pAb and MPO) as normalized average (see Fig. 3). Figure 3 compares the composite and immunocomplex spectra, and for ease of visualization the spectra was divided into 3 sections and presented as Fig. 3a, b, and c. The ability to differentiate such subtle differences without extensive data analysis procedures demonstrates the simplicity of the approach and the sensitivity of the SERS method in differentiating the binding interactions of an immunocomplex from unbound antigen/antibody pair. Relative standard errors (RSE) calculated $(n=80)$ at peak positions that are unique for the immunocomplex are shown in Table 3. RSE at the new peak positions varied significantly, with a minimum deviation (18\%) at $1465 \mathrm{~cm}^{-1}$. We attribute these variations to possible orientation differences of the Au particles. It is well established that the amplification factor of the SERS substrate largely depends on the crystal facets to which the molecules are absorbed. Recently Yu et al employed SERS for studying the effect of charge on the orientation of cytochrome c [34] and concluded that the molecules have random orientation on a bare Au nanohole surface. In the present study, it is highly probable that orientation and the crystal facet of the $\mathrm{Au}$ particles were totally random. Since the SERS data was collected over a large surface area compared to the size of a cluster of particles it would be important to identify the influence of the crystal orientation on the obtained SERS data, especially on the new peaks. We believe it is possible to minimize these variations by improving the homogeneity of the SERS substrate using the methods proposed by Liu et al [27].

Table 3. Relative standard error at peak positions identified as unique to immunocomplex.

\begin{tabular}{cc}
\hline Peak Position $\left(\mathbf{c m}^{-\mathbf{1}}\right)$ & Relative Standard Error $(\%)$ \\
\hline 870.6798 & 48.0 \\
968.7625 & 48.0 \\
1109.126 & 62.0 \\
1145.536 & 51.0 \\
1464.528 & 18.0 \\
1500.479 & 30.0 \\
\hline
\end{tabular}

\section{CONCLUSIONS}

Our results demonstrate that binding between an antigen (MPO) and its antibody gives rise to unique peaks absent in the compsoite spectra, derived by mere addition of the respective MPO and MPO-Ab Raman signatures. A valid question is how universal these peaks are and if one should expect to find identical or similar peaks upon binding of any antigen to its antibody. Data on the Raman of $\mathrm{IgG}$ antibodies have demonstrated that all IgG Raman spectra are very similar, while antigen Raman spectra depend on their particular structures. For classes of antigen / IgG antibodies where the binding interactions resemble the MPO/MPO-Ab binding, one would expect to find 
similar peaks, possibly shifted but in the vicinity of the peaks we have identified. However, for binding pairs where the interaction sites are different chemical entities, totally different peaks would arise. A systematic study of such classes of antigen/Ab pairs would be very useful to create a library of practical significance to many scientific studies.

In summary, we investigated the potential application of SERS in differentiating the bound immunocomplex of an antigen and its antibody from the unbound complex and its components using myeloperoxidase as the model antigen. Obtained results indicate that the SERS spectrum of the immunocomplex is different from that of its parent antigen or antibody, and it is possible to identify conformational changes due to immunocomplex formation. Furthermore, the smallest RSE $(18 \%, \mathrm{n}=80)$ at $1465 \mathrm{~cm}^{-1}$ in the SERS spectra of the immunocomplex supports the notion that further investigation at this particular vibrational mode could provide valuable information towards application of SERS in understanding the changes that occur at a molecular level during binding interactions in biomolecules.

The authors wish to express their gratitude for the expert advice and training provided by Dr. Zhorro Nikolov, Director of the Centralized Research Facilities at Drexel University. This work was partially supported by the W. M. Keck Institute for Attofluidics at Drexel University.

\section{Received 5 March 2010; accepted 30 March 2010; published online 27 April 2010.}

\section{References}

1. S. Nie and S. R. Emory, Science 275, 1102 (1997). doi:10.1126/science. 275.5303 .1102

2. M. Fleischmann, P. J. Hendra and A. J. McQuillan, Chem. Phys. Lett. 26, 163 (1974). doi:10.1016/0009-2614(74) $\underline{85388-1}$

3. D. L. Jeanmaire and R. P. Van Duyne, J. Electroanal. Chem. 84, 1 (1977). doi:10.1016/S0022-0728(77)80224-6

4. M. G. Albrecht and J. A. Creighton, J. Am. Chem. Soc. 99, 5215 (1977). doi:10.1021/ja00457a071

5. J. Hu, Z. Wang and J. Li, Sensors 7, 3299 (2007). doi:10.3390/s7123299

6. M. Suzuki, Y. Niidome, Y. Kuwahara, N. Terasaki, K. Inoue and S. Yamada, J. Phys. Chem. B 108, 11660 (2004). doi:10.1021/jp0490150

7. P. C. Lee and D. Meisel, J. Phys. Chem. 86, 3391 (1982). doi: $10.1021 / \mathrm{j} 100214 \mathrm{a} 025$
8. K. Kneipp, R. R. Dasari and Y. Wang, Appl. Spectrosc. 48, 951 (1994). doi:10.1366/0003702944029776

9. K. Stubenrauch, U. Wessels, R. Vogel and J. Schleypen, Analy. Biochem. 390, 189 (2009). doi:10.1016/j.ab.2009. $\underline{04.021}$

10. H. W. Zhao, C. Z. Huang, L. P. Wu, S. F. Shen and Z. H. Qin, Anal. Lett. 42, 1495 (2009). doi:10.1080/000327 $\underline{10902961099}$

11. K. Kneipp, A. S. Haka, H. Kneipp, K. Badizadegan, N. Yoshizawa, C. Boone, K. E. Shafer-Peltier, J. T. Motz, R. R. Dasari and M. S. Feld, Appl. Spectrosc. 56, 150 (2002). doi:10.1366/0003702021954557

12. L. J. Goeller and M. R. Riley, Appl. Spectrosc. 61, 679 (2007). doi:10.1366/000370207781393217

13. A. K. Kalkan and S. J. Fonash, Appl. Phys. Lett. 89, 233103 (2006). doi:10.1063/1.2399369

14. E. J. Bjerneld, Z. Foldes-Papp, M. Kall and R. Rigler, The J. Phys. Chem. B 106, 1213 (2002). doi:10.1021/jp $\underline{012268 \mathrm{y}}$

15. T. E. Rohr, T. Cotton, N. Fan and P. J. Tarcha, Anal. Biochem. 182, 388 (1989). doi:10.1016/0003-2697(89) 90613-1

16. M. L. Zhang, C.Q. Yi, X. Fan, K. Q. Peng, N. B. Wong, M. S. Yang, R. Q. Zhang and S. T. Lee, Appl. Phys. lett. 92, 043116 (2008). doi:10.1063/1.2833695

17. S. R. Hawi, S. Rochanakij, F. Adar, W. B. Campbell and K. Nithipatikom, Anal. Biochem. 259, 212 (1998). doi:10.1006/abio.1998.2661

18. X. Dou, Y. Yamaguchi, H. Yamamoto, S. Doi and Y. Ozaki, J. Raman Spectrosc. 29, 739 (1998). doi:10.1002/(SICI) 1097-4555(199808)29:8<739::AID-JRS289>3.0.CO;2-S

19. J. Merlie, D. Fagan, J. Mudd and P. Needleman, J. Biol. Chem. 263, 3550 (1988).

20. S. Kimura and M. Ikeda-Saito, Protein. Struct. Funct. Genet. 3, 113 (1988). doi:10.1002/prot. 340030206

21. P. M. Marie-Madeleine CALS, Ghislaine BRIGNON, Patricia ANGLADE and Bruno Ribadeau DUMAS, Eur. J. Biochem. 198, 733 (1991).

22. J. Zeng and R. E. Fenna, J. Molecular Bio. 226, 185 (1992). doi:10.1016/0022-2836(92)90133-5

23. S. S. Sibbett and J. K. Hurst, Biochem. 23, 3007 (1984). doi:10.1021/bi00308a025

24. G. Frens, Nature: Physical Science 241, 20 (1973).

25. W. Haiss, N. T. K. Thanh, J. Aveyard and D. G. Fernig, Anal. Chem. 79, 4215 (2007). doi:10.1021/ac07 02084

26. S. H. Park, J. H. Im, J. W. Im, B. H. Chun and J. H. Kim, 
Microchemical Journal 63, 71 (1999). doi:10.1006/mchj. $\underline{1999.1769}$

27. F. M. Liu and M. Green, J. Mater. Chem. 14, 1526 (2004). doi: $10.1039 / \mathrm{b} 400488 \mathrm{~b}$

28. G. T. Babcock, R. T. Ingle, W. A. Oertling, J. C. Davis, B. A. Averill, C. L. Hulse, D. J. Stufkens, B. G. J. M. Bolscher and R. Wever, BBA-Protein Struct. M. 828, 58 (1985).

29. G. J. Puppels, H. S. Garritsen, G. M. Segers-Nolten, F. F. de Mul and J. Greve, Biophys. J. 60, 1046 (1991). doi:10.10 16/S0006-3495(91)82142-7

30. N. M. Sijtsema, Thesis, University of Twente, 1997.
31. C. Otto, N. M. Sijtsema and J. Greve, Eur. Biophys. J. 27, 582 (1998). doi:10.1007/s0024900 50169

32. S. D. Zbylut and J. R. Kincaid, J. Am. Chem. Soc. 124, 6751 (2002). doi:10.1021/ja01 2578u

33. A. M. Ahern and R. L. Garrell, Langmuir 7, 254 (1991). doi:10.1021/1a00050a009

34. Q. M. Yu and G. Golden, Langmuir 23, 8659 (2007). doi:10.1021/la7007073

35. S. Brogioni, A. Feis, M. P. Marzocchi, M. Zederbauer, P. G. Furtmüller, C. Obinger and G. Smulevich, J. Raman Spectrosc. 37, 263 (2006) doi:10.1002/jrs.1442 